\title{
MDRTB Then, XMDRTB Now and Then ......... Totally
}

\author{
Ajaz Nabi Koul, M.D., F.R.C.P \\ Department of Internal Medicine, SKIMS, Srinagar, Kashmir.
}

\section{A B S T R A C T}

Multi drug resistant tuberculosis has come up as a separate disease meriting different diagnostic and therapeutic options from conventional tuberculosis. Worldwide there has been considerable increase in MDRTB cases to 60,000 and less than $5 \%$ of them get management as desired by various infectious disease bodies. Another entity titled XMDRTB has of late assumed significant proportion of tuberculosis cases which is steaming into a new health related inferno.

Some sporadic cases of tuberculosis have come up which are completely resistant to all anti-tubercular drugs, that should be an area of concern for all healthcare givers. JMS 2016; 19(1):12-17

Key words: Tuberculosis, MDRTB, XMDRTB.

Multidrug-resistant tuberculosis is defined as disease caused by Mycobacterium tuberculosis that are at least resistant to isoniazid and rifampicin and extensively drugresistant tuberculosis refers to disease caused by multidrugresistant strains that are also resistant to treatment with any fluoroquinolone and aminoglycosides. MDR tuberculosis was a serious health concern for past two decades and presently XDR tuberculosis is a serious threat to the achievements made worldwide in controlling the menace of morbidity and mortality associated with TB. ${ }^{1}$

As per recent estimate of WHO regarding trends of tuberculosis worldwide, 8.7 million cases and 1.4 million deaths due to tuberculosis has been seen globally in 2012 . The figures seem to be reasonably under control and trends are healthy for effective control of tuberculosis. At the same time there has been substantial increase in MDRTB cases. A total of $60 \mathrm{k}$ cases have been reported from 58 countries, which is having upward trend. National programs are failing to diagnose and treat MDR tuberculosis. Worldwide 30,000

\section{Correspondence}

Dr. Ajaz Nabi Koul, M.D.,

Associate Professor, Internal Medicine, SKIMS, Srinagar AssociateEditor, JournalofMedical

Sciences, SKIMS cases of MDR tuberculosis were reported to the World Health Organization (WHO) in 2008, of which less than one fifth were managed according to international guidelines. Still majority of the cases probably are not diagnosed or, if diagnosed are mismanaged. The problem still remains at large that MDRTB is undiagnosed, untreated, ill-treated, under reported. The health care professionals, international bodies and administrative hierarchy does not recognize MDRTB a disease other than $\mathrm{TB}^{2}$.

In some countries like Botswana and Korea the incidence of MDRTB is fast increasing although the incidence of drug susceptible tuberculosis is steady. In some countries like US, west Europe the incidence of tuberculosis in decreasing. As per the recent data from WHO the global burden of tuberculosis is stable to decreasing trend depending on geographical variation. The same is not true about MDRTB where the burden is increasing globally every passing year. At present in four regions like India, China, South Africa, Brazil and Sub Saharan Africa the burden of MDRTB is on rise. ${ }^{3,4,5}$

The WHO-recommended Stop TB Strategy advocates the early detection and effective observed therapy for treatment and eradication of tuberculosis. The DOTS programme 
encourages the political and administrative coordination between various medical and voluntary organizations to plan and act for culminating the disease load of tuberculosis worldwide. It was due to coordination between all member countries of WHO that between 1995 and 2008, a total of 36 million people were treated successfully with the use of the DOTS approach, and it was estimated that about 6 million lives were saved. There are guidelines for management of drug-resistant TB but till date planning, funding, and implementation of these recommendations is falling short of goals which were set by WHO and infectious disease bodies worldwide. ${ }^{6}$

After thorough analysis of the global concern over the rising cases of MDRTB the Bill and Melinda Gates foundation in collaboration with the Chinese ministry of health in 2009 organized WHO ministerial meeting to discuss the factors leading to increase global burden of MDRTB. Many factors were studied and some recommendations were put forward to control the increase in MDRTB cases. In the meeting it was laid with emphasis that better coordination, ample funding, research is the need of hour for diagnosis and treatment of MDRTB. There is urgent need for early suspecting of MDRTB followed by drug sensitivity testing and effective drug therapy.

Resistance to anti-tuberculosis agents has been studied since the 1940s. The blueprints for containing MDR tuberculosis were laid out in the clinical literature and in practice, several settings more than 20 years ago ${ }^{2}$. Yet today, barely $0.5 \%$ of persons with newly diagnosed MDR tuberculosis worldwide receive treatment that is considered the standard care in the United States. Following measures need to be undertaken in step wise fashion to limit the spread of MDRTB.

\section{Education about MDRTB}

The need of the hour is that every health care individual should be sensitized with a new disease MDRTB, with its clinical course, presentations, suspicion of its occurrence, importance of drug sensitivity testing, appropriate antituberculosis drug combination, adequate treatment duration, contact tracing, complication recognition and its approach lastly in recognition of predisposing factors. In addition to sensitize health care workers it is imperative to make mass awareness programme for common public especially for the family of the affected regarding the need to adherence of drug regimens.

\section{Financial Strategy}

The cost of management of tuberculosis, at individual or state level remains a big challenge for all the nations in the world especially the economically underprivileged countries. On top of this scenario the trouble for drug resistant tuberculosis remains more deep. Not only the treatment of such patients is costly but as the diagnostic challenges widen the cost for isolation and studying the sensitivity patterns also surmounts huge monetary debts. It has been estimated that management of MDRTB ranges from 4000 to 5000 dollars, till completion of treatment. Such financial burdens have outreaching impact in developed countries like US, one can imagine the crunch it will have in countries like Rawanda, Chile and Thailand.

Even before universal health coverage is achieved, immediate steps can be taken to reduce catastrophic health expenditures for patients with tuberculosis and their households.'. These steps include decentralization of services to reduce the indirect costs that patients seeking care incur, provision of patient incentives and social support to promote adherence to treatment, with subsidization of care provided in the private sector that is in line with guidelines from national tuberculosis programs.

\section{Training and Motivation of Professionals}

It has been estimated that half of the patients of tuberculosis are being managed by those health care professionals who are not trained in respective national tuberculosis programmes or have no qualified experience to manage MDRTB cases.

Nonetheless, only a fraction of the tuberculosis cases diagnosed by practitioners outside the public sector are registered with or referred to national tuberculosis programs. ${ }^{8,9}$ These approaches should therefore be scaled up and applied to the prevention and management of MDR tuberculosis as well.

Training, technical and financial support, and the supervision for prompt and National tuberculosis programs need to play a leading role and provide guidelines, effective management of tuberculosis and particularly MDRTB by International Standards for TB $\mathrm{Care}^{10}$. Professional associations need to act as intermediaries between national tuberculosis programs and private providers. Non-national programmes have done a commendable job in various countries, which has been upholded by reputed infectious diseases groups and in particular WHO has held them as 
benchmarks.

Some regulation may be necessary. In some countries with a high burden of tuberculosis, reporting of tuberculosis is not mandatory by law. In India till May 2012 it was not imperative to report diagnosed or suspected case of tuberculosis but presently as per legislation whether the patient belongs to private or government sector needs to be notified. In some countries where notification is required, systems have not been established to ensure that the requirement is applied. Case notification for both tuberculosis and MDR tuberculosis must be made mandatory by legislation.

\section{Optimizing Disease Management and Care}

Transmission of drug-resistant tuberculosis occurs in the community, as indicated by the high frequencies of MDR tuberculosis among previously untreated patients in some countries. In most countries with limited resources, patients with MDR or XDR tuberculosis must complete two unsuccessful courses of treatment with first-line antituberculosis drugs before being eligible for treatment with second-line drugs. Moreover, in many countries, treatment of MDR tuberculosis is started only after the diagnosis has been confirmed, a process that takes months when conventional methods are used. As a result, persons with infectious MDR or XDR tuberculosis remain in the community for long periods of time. Prompt diagnosis and treatment of tuberculosis and MDR tuberculosis can keep the case reproduction number of MDR strains below their replacement rate and perhaps even below that of non-MDR strains. $^{11,12}$

Outbreaks of MDR tuberculosis have occurred in hospitals, and patients with tuberculosis who are hospitalized have a higher risk of acquiring MDR tuberculosis than do those who are treated as outpatients. Treating MDR tuberculosis in a hospital is more expensive than doing so on an ambulatory basis. Hospital treatment is also more socially and economically disruptive for most patients. In addition, the number of hospital beds may become insufficient as countries expand treatment for MDR tuberculosis. Despite the complexities involved in caring for patients with MDR tuberculosis, including lengthy therapy with poorly tolerated drugs, clinic-based or community-based care has proved to be feasible and effective in several countries, including Nepal and Peru. However, the effectiveness of outpatient care depends on the availability of primary care facilities, qualified health care workers, and social support networks to promote adherence to treatment. Countries need to select the model of care that is right for them, taking into account the personal rights and needs of patients and communities. $^{13}$

\section{Upgradation of Diagnostic Facilities}

Appropriate and prompt diagnostic testing including drug sensitivity testing (DST) is hallmark for success against tuberculosis with added emphasis on drug resistant tuberculosis. It has been estimated that $<3 \%$ cases have undergone DST in tuberculosis in 2012.

Rapid molecular tests for MDR tuberculosis are available. Automated rapid test for rifampicin resistance holds promise for easier detection of MDR tuberculosis The implementation of this and other rapid tests, like line probe assays for diagnosing drug resistance especially in countries with a high prevalence of concurrent HIV infection and MDR tuberculosis, can prevent fatal delays in detection. Use of the new molecular technologies offers one of the best avenues for improving overall diagnostic capacity in the laboratory. At present, however, the adoption of the new rapid tests will not eliminate the need for conventional drugsusceptibility testing with the use of solid or liquid culture. Conventional susceptibility testing is required to determine susceptibility to drugs other than rifampicin and isoniazid. All the countries need to expand laboratory capacity and introduce the new rapid tests, targeting drug-susceptibility testing. The more time is consumed in detection of tuberculosis and its susceptibility pattern more chances of drug resistance is going to be escalated ${ }^{20,21}$.

\section{Drug Supply}

It has been estimated that substandard and spurious drug market for tuberculosis has expanded in past two decades. As per green light committee criteria of WHO only those manufacturers of ATT drugs be allowed to supply the drugs who meet the criteria laid down by who screening committee. Despite many corrigendum by WHO only 70 countries have followed the norms laid for supply of effective medicines to these patients.

There should be proper inspection and quality control of these drugs, in addition all the countries should limit over the counter sale and dispensing of these life reconstructing drugs. 


\section{Drug Research}

There is long felt need to expand the armamentarium of drugs needed for management of tuberculosis patients. It is high time to find alternative drug regimes for tailoring the management strategies for MDRTB.

During past decade variety of conventional molecules were found to have anti-tubercular activity. It has been laid to individual treating physicians to tailor the management protocol for management of individual MDRTB patient. The area is more mercurial for XDRTB and may be gloomy for those patients who are resistant to almost all antitubercular drugs. Although so much dismal may not be the scene but in an odd patient a clinician is faced with a situation where all the guns become trigger less.

\section{Domicillary Treatment V/S Hospital Treatment}

Every action has pros and cons. Hospital treatment enhances detection of complications related to disease or treatment, supervised treatment, counseling, peer pressure, changing odd regimens etc. as against domiciliary treatment which need more and more commitment from individual to family and community. In addition the spread of cross infection in hospitals remains at low ebb constrained by infection control regulations.

\section{Call For Action Not Words}

Potential weaknesses in practices and guidelines for management and control of MDR tuberculosis and XDR tuberculosis have been identified and are being assessed at the global level. 25 high burden countries have been identified and the regulatory bodies of these respective countries have made effective policies in consultation with national control programme to coordinate diagnosis and treatment strategies for resistant tuberculosis. More than 500 million dollars is needed to combat the menace of MDRTB in next 5 years. The treatment and control of MDR tuberculosis requires action beyond national tuberculosis control programs, sometimes in the political environment outside the health care system.

\section{Is There A MDRTB Or XDRTB Policy or where are we 9000 Years Ago?}

Drug resistance is well established as inevitable once antibiotics are used. The issue of the MDR tuberculosis pandemic are largely man-made. The contours of global efforts against tuberculosis have always been mediated by both biologic and social determinants, and the reasons for the divergence in the rates of tuberculosis and drug resistance between rich and poor countries are biosocial. As case rates dropped in wealthy countries, funding for research and implementation programs dried up, even though tuberculosis remained the world's leading infectious killer of young adults throughout the 20th century. These complexities, which now range from pan-resistant tuberculosis to undiagnosed pediatric disease, account for more than a trivial fraction of the 9 million new cases of tuberculosis and the almost 2 million deaths from this disease that occur around the globe each year.

New diagnostics and therapeutics are urgently needed; most of the methods used currently were developed decades ago. Today, we have rapid nucleic acidbased tests for drugresistant tuberculosis, sound models for laboratory expansion and for treatment delivery, and several drug candidates in the pipeline. To tackle tuberculosis, we also need an equity plan that takes seriously the biosocial complexity of a lethal airborne infection that has stalked us for centuries. The global AIDS effort of the past decade has shown how much can be accomplished in global health when effective diagnosis and care are matched with funding and political will. Stinting on investments or on bold action against tuberculosis in all its forms will ensure that it remains a leading killer of people living in poverty in this decade and the next.

\section{The controversy of TDRTB}

This debate got clicked at Hinduja Hospital last year where a patient with tuberculosis was diagnosed who has a strain totally resistant to all first and second line drugs. As this case was reported in literature an international urge came into play that we are faced with complete helplessness against tuberculosis. As the discussions and counter discussions flowed from lancet to European thoracic society and international league against infectious diseases ${ }^{14}$. The nutshell came that it will dismay hopes to conquer tuberculosis, and such terms like totally resistant TB should be done away ${ }^{15,16,17}$. But at the same time it is imperative for all health care bodies, professionals and administrators to reconsider that a practical situation exists when a clinician gets locked in, with what to treat this TB. A spark which seems to be distant today may herald a disaster in generation to follow. We need to recognize that it is a new disease nad not TB. ${ }^{(18,19}$

\section{Practice Points ..... Need of the Hour}

1. Drug sensitivity testing for Tuberculosis should be a standard practice, with conventional methods being 
corner stone but time consuming. The time lag can be bridged by rapid diagnostic methods.

2. Appropriate drug regimes must be tailored for individual patients considering the sensitivity patterns, co morbid conditions, smear positivity versus negativity, site of affliction.

3. Role of alternate effective and compliant therapy in case of drug intolerance, drug toxicity or drug unavailability.

4. Maintenance of brazen difference between TB, MDRTB, XDRTB and so on to decrease chances of development of drug resistance while patient is on treatment.

5. Surgical options should always be on cards in those patients where the TB needs removal of disease burden. It not only helps to remove unwanted debris but also limits the spread of disease and better drug penetration into tissues.

6. A healthy coordination among national programmes and non- national programs.

7. Mass awareness programmes, community based education programmes, involvement of print and electronic media.

8. Need based education and involvement of administrators and political agencies.

9. Continous education programmes for health care professionals who are directly and indirectly involved in management of tuberculosis.

10. Financial generation for prompt, effective and regime based uninterrupted supply of second or third line regimes.

11. Incentive wise involvement of pharmaceuticals in manufacturing of second and third line anti tubercular drugs.

\section{REFERENCES}

1. Global tuberculosis report

2012. Geneva: World Health Organization.

2. Tuberculosis MDR-TB and XDR-TB: 2011 progress report. Geneva: World Health Organization, 2012.

3. Surveillance report: tuberculosis surveillance and monitoring in Europe 2012 Geneva: World Health Organization.

4. Abubakar I, Lipman M, Anderson C, Davies P, Zumla
A. Tuberculosis in the UK Time to regain control. BMJ 2011;343:d4281.

5. Verver S, Warren RM, Beyers N, et al. Rate of reinfection tuberculosis after successful treatment is higher than rate of new tuberculosis. Am J Respir Crit CareMed 2005;171:1430-5.

6. Frieden TR, Munsiff SS, Ahuja SD.Outcomes of multidrug-resistant tuberculosis treatment in HIVpositive patients in New York City, 1990-1997. Int J TubercLung Dis 2007;11:116.

7. Cain KP, McCarthy KD, Heilig CM, et al. An algorithm for tuberculosis screening and diagnosis in people with HIV. N Engl J Med 2010;362:707-16.

8. McNerney R, Maeurer M, Abubakar I, et al. Tuberculosis diagnostics and biomarkers: needs, challenges, recent advances, and opportunities. J Infect Dis 2012;205:Suppl 2:S147-S58.

9. von Reyn CF, Horsburgh CR, Olivier KN, et al. Skin test reactions to Mycobacterium tuberculosis purified protein derivative and Mycobacterium avium sensitin among health care workers and medical students in the United States. Int J Tuberc Lung Dis 2001;5:1122-8.

10. Hopewell PC, Pai M, Maher D, Uplekar M, Raviglione MC. International standards for tuberculosis care. Lancet Infect Dis 2006;6:710-12

11. Policy guidance on drug-susceptibility testing (DST) of second-line antituberculosis drugs. Geneva: World Health Organization, 2008.

12. Ling DI, Zwerling AA, Pai M. Geno- Type MTBDR assays for the diagnosis of multidrug-resistant tuberculosis: a meta-analysis. Eur Respir J 2008;32:1165-74.

13. Rapid implementation of the Xpert MTB/RIF diagnostic test: technical and operational 'how-to': practical considerations. Geneva: World Health Organization, 2011

14. Udwadia ZF, Amale RA, Ajbani KK, Rodrigues C. Totally drug-resistant tuberculosis in India. Clin Infect Dis 2012;54: 579-81.

15. "Totally Drug-Resistant TB": a WHO consultation on the diagnostic definition and treatment options. Geneva: World Health Organization (http://www.who. int/tb/challenges/xdr/Report_Meeting_totallydrug resistantTB_032012.pdf). 
16. Nathanson E, Nunn P, Uplekar M, et al. MDR tuberculosis critical steps for prevention and control. $\mathrm{N}$ Engl J Med 2010;363:1050-8.

17. Lawn SD, Zumla AI. Tuberculosis. Lancet 2011;378:57-72.

18. Tuberculosis: clinical diagnosis and management of tuberculosis, and measures for its prevention and control. London: National Institute for Health and Clinical Excellence, 2011 (http://www.nice.org.uk/ nicemedia/live/13422/53642/53642 .pdf).

19. Hopewell PC, Pai M, Maher D, Uplekar M, Raviglione MC. International standards for tuberculosis care. Lancet Infect Dis 2006;6:710-25.
20. Boehme CC, Nabeta P, Hillemann D, et al. Rapid molecular detection of tuberculosis and rifampin resistance. N Engl J Med 2010;363:1005-15.

21. Lawn SD, Kerkhoff AD, Vogt M, et al. Characteristics and early outcomes of patients with Xpert MTB/RIFnegative pulmonary tuberculosis diagnosed during screening before antiretroviral therapy. Clin Infect Dis 2012;54:1071-9. 